\title{
Testing and Estimating the Non-Disjunction Fraction in Meiosis I using Reference Priors
}

\author{
Rosangela H. Loschi", João V. D. Monteiro, Gustavo H. M. A. Rocha, \\ and Vinicius D. Mayrink \\ Departamento de Estatística - ICEx, Universidade Federal de Minas Gerais, Av. Antonio Carlos 6627, \\ Pampulha, 31270-901 Belo Horizonte, MG, Brazil \\ Received 01 February 2007, revised 27 March 2007, accepted 26 April 2007 \\ Summary \\ In this paper we analyze the fraction of non-disjunction in Meiosis I assuming reference (non-informa- \\ tive) priors. We consider Jeffreys's approach to built a non-informative prior (Jeffreys's prior) for the \\ fraction of non-disjunction in Meiosis I. We prove that Jeffreys's prior is a proper distribution. We \\ perform Monte Carlo studies in order to compare Bayes estimates obtained assuming Jeffreys's and \\ uniform priors. We consider full Bayesian significance test (FBST) and Bayes factor (BF) for testing \\ precise hypothesis on the fraction of non-disjunction in Meiosis I. The ultimate goal of this paper is to \\ compare these two test procedures through simulation studies using both prior specifications. An appli- \\ cation to Down Syndrome data is also presented.
}

Key words: Bayes factor; Full Bayesian significance test; Non-informative priors; Trisomy.

\section{Introduction}

Chromosomal anomalies can be structural or numerical. The numerical chromosomal anomalies, named aneuploidies, are common causes of mental retardation, pregnancy losses and fetal death in humans. One of the best known aneuploidies is the trisomy of chromosome 21, which produces a phenotype known as Down syndrome. Aneuploidies, in general, arise from sporadic error in the chromosomal segregation during the meiotic process, named a non-disjunction. Non-disjunctions can occur in the first (meiosis I) or second (meiosis II) division in the meiotic process. Despite its importance, little information about the genetic and environmental causes of aneuploidies is available. The determination of the rate of non-disjunction in chromosomal segregation, which takes place in meiosis I in each chromosome, is useful to identify possible factors (as, for example, geography, nutrition, age, reproductive practices, etc.), which generate such numerical chromosomal anomalies. For example, in the trisomy of chromosome 21, there is evidence that the rate of non-disjunction increases with the age of the mother (see Pena, 1998). Moreover, the increase in the rate of non-disjunction in meiosis II is higher than for meiosis I if the mother's age is between 35 and 39 years. For sexual chromosomes, however, high mother age influences only the fraction of non-disjunction in meiosis I. See Franco et al. (2003) for details.

The meiotic cellular division consists of the DNA replication followed by two cell divisions generating haploid cells. In meiosis I, aneuploidies are due to the non-disjunction of homologous chromosomes which remain unite and migrate to the same cell. In this cases, when the abnormal cell crosses with a normal gamete we can observe one, two or three different alleles depending on the genetic

\footnotetext{
* Corresponding author: e-mail: loschi@est.ufmg.br, Phone: +55 313499 5939, Fax: +55 3134995924
} 


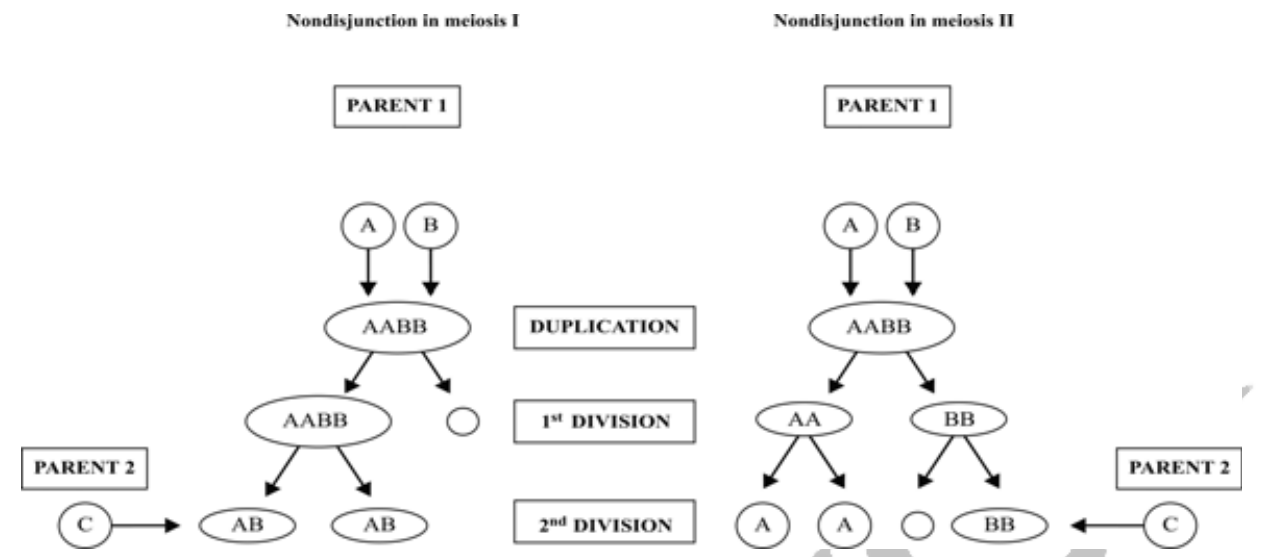

Figure 1 Meiosis Non-disjunction scheme.

characteristics of the parents. In meiosis II, aneuploidies are a consequence of the non-disjunction of sister chromatids which remain unite and migrate to the same cell. In this case, the two alleles will be the same and it is impossible to have a three peaks pattern. In order to make easy the understanding of the non-disjunction in meiotic process, Franco et al. (2003) provides the scheme presented in Figure 1, where $A$ and $B$ denote the alleles of a particular microsatellite present in parent 1 and $C$ is the allele in parent 2 that will joint to the alleles of parent 1 in the fertilization process.

The diagnosis of the syndrome is done by employing a polymerase chain reaction (PCR) based approach. It is possible to type microsatellites located near the chromosomal centromere (to avoid problems due to recombination) with the PCR using primers designed from the unique sequence DNA flanking the tandem repeat arrays, followed by quantitative analysis by computer-assisted laser densitometry (Pena, 1998). Microsatellite loci will be directly informative whenever they show more than one allele peak. Trisomic patients will display, in informative microsatellite loci, three fragment peaks of equal intensity, two fragments at an average $2: 1$ dosage or one individual fragment. The relative proportion of the three cases will depend on the type of non-disjunction, although the heterozygosity level is also important. For a three-allele pattern to emerge it is necessary for the non-disjunction to occur in the first meiotic division, the mother to be heterozygous at the relevant locus and the allele carried by the sperm to be different from the two maternal alleles (that is, $A, B$ and $C$ in Figure 1 are all different alleles). A two-allele pattern is observed either in the first or second meiosis division, depending on the combination of the chromosomes transmitted by the parents. One peak pattern occurs if the parents are homozygous for the allele inherited. See details in Hassold and Jacobs (1984).

Many proposed methods to estimate $\phi$ (given that a non-disjunction occurs, $\phi$ denotes the conditional probability that a non-disjunction occurs in the first division of meiosis) in trisomies take into account the comparative studies of pericentromeric DNA polymorphic markers from affected children and their parents (Hassold and Hunt, 2001). More recently, some estimates of $\phi$ were obtained considering only the information extracted from the DNA of affected children through PCR (see Franco et al. (2003), Nicolaidis and Peterson (1998), Savage et al. (1998), Yoon et al. (1996), Koehler et al. (1996), Griffin (1996), Peterson et al. (1992), Zaragosa et al. (1994) and many others). For trisomies, Franco et al. (2003) propose a model for the number of peaks in a polymorphic locus which depends on the fraction $\phi$ of meiosis I non-disjunction. This model does not take the parental information into account. The model proposed by Franco et al. (2003) is very useful since the necessity of using the parental information can make research more cumbersome and disable the use of archive material, which could be a problem in rare aneuploidies. Assuming that the relative frequency of each allele is known and that the hypothesis of Hardy-Weinberg equilibrium (Hartl and Clark, 1997) is verified for 
the population, Franco et al. (2003) establish that the number of individuals with one, two or three peaks, given $\phi$, can be modeled by a multinomial distribution. Franco et al. (2003) present the maximum likelihood (ML) estimator and bootstrap intervals for $\phi$. Later on, Loschi and Franco (2006) extended such an analysis including prior information into the problem. These authors compare Bayes and ML estimators through Monte Carlo studies concluding, as expected, that posterior modes and ML estimates are similar if the uniform prior distribution is assumed to describe the initial uncertainty about $\phi$. Loschi and Franco (2006) also concluded that Bayes estimators provide better estimates than ML estimator if fair informative prior distributions for $\phi$ are considered.

Frequently, the number of trisomic patients is small compromising the use of asymptotic theory (see Franco et al. (2003), for instance). Moreover, in many circumstances, the initial information about $\phi$ can also be rare - which can occur in rare aneuploidies, for example - compromising the use of informative priors. The usual criticism against Loschi and Franco's (2006) approach is that they do not consider reference priors. It is well known that prior specifications permit to incorporate scientific hypotheses into the analysis and, consequently, their use allows to handle complex problems and situations in which little sample information is available. In such situations, reference (or non-informative) priors are frequently used to describe prior uncertainty about the parameter. There are several methods to construct non-informative priors. In this paper, we consider Jeffreys's approach (Jeffreys, 1961) to obtain a non-informative prior for $\phi$ (Jeffreys's prior) since it is extensively used in the literature. According to Migon and Gamerman (1999), the idea behind Jeffreys's prior is to provide as little prior information as possible, relative to the sample information. We prove that Jeffreys's prior is a proper distribution. We also perform a Monte Carlo study in order to compare the Bayes estimates assuming two non-informative prior specifications: Jeffreys's and uniform priors. According to Barros and Franco (2002) one of the main interest is to verify if, for the Brazilian individuals with Down syndrome, the fraction of non disjunction in meiosis I is 0.68 . Due to the fact that chromosome trisomies are rare events and, consequently, few information about it are available, Barros and Franco (2002) employ the bootstrap confidence intervals to test precise hypothesis about $\phi$. The ultimate goal of this paper is to introduce two Bayesian procedures for testing hypothesis about $\phi$ : full Bayesian significance test (FBST) (Pereira and Stern, 1999) and Bayes factor (BF) (see Bernardo and Smith (1994), for instance). To test precise hypothesis, BF can lead to Lindley's paradox (Tsao, 2006). FBST was introduced in literature in order to avoid such a problem. To perform the FBST we need only the knowledge of the parameter space represented by its posterior distribution and do not need additional assumptions, such as a positive prior probability for the precise hypothesis (Madruga et al. 2003). Then, it is also our goal to compare these two hypothesis test procedures through simulation studies. We consider both prior specifications and only precise hypothesis about $\phi$. Finally, we analyze a sample of Brazilian individuals with Down syndrome in order to illustrate the methodologies. The results are compared with those found in literature.

The paper is organized as follows: Section 2 presents the likelihood function and Jeffreys's prior for $\phi$. Bayes factor and the evidence of null hypothesis are obtained to test precise hypotesis about $\phi$. Section 3 presents Monte Carlo studies to compare Bayes estimates for $\phi$ as well as BF and FBST, using Jeffreys's and uniform prior specifications. In Section 4, we analyze the data from Brazilian patients with trisomy of chromosome 21. Finally, in Section 5 we draw some conclusions.

\section{Statistical Model}

In this section we obtain Jeffreys's prior for $\phi$ and describe some techniques for testing and estimating $\phi$ from the Bayesian point-of-view.

\subsection{Likelihood of $\phi$}

To construct the likelihood function of $\phi$, Franco et al. (2003) assume that the hypothesis of HardyWeinberg equilibrium (Hartl and Clark, 1997) is verified for the population. 
Denote by $Y_{l}$ the number of individuals with $l$ peaks pattern, $l=1,2,3$, to be observed in a sample of $n$ trisomic individuals and denote by the random vector $\left(Y_{1}, Y_{2}, Y_{3}\right)$. Let $p_{i}, i=1, \ldots, m$, be the relative frequency of the allele $i$ in a multiallelic locus of microsatellites. Denote by $\phi, \phi \in[0,1]$, the conditional probability of the non-disjunction taking place in meiosis I, given that an error of nondisjunction occurred. Given $\phi$, Franco et al. (2003) show that the random vector $\boldsymbol{Y}$ has a multinomial distribution with parameters $n, \theta_{1}(\phi)>0, \theta_{2}(\phi)>0$ and $\theta_{3}(\phi)>0$, denoted by $\boldsymbol{Y} \sim \operatorname{Mult}\left(n, \theta_{1}(\phi)\right.$, $\left.\theta_{2}(\phi), \theta_{3}(\phi)\right)$, which has probability function given by:

$$
P(\boldsymbol{Y}=\boldsymbol{y} \mid \phi)=\frac{n !}{y_{1} ! y_{2} ! y_{3} !}\left[\theta_{1}(\phi)\right]^{y_{1}}\left[\theta_{2}(\phi)\right]^{y_{2}}\left[\theta_{3}(\phi)\right]^{y_{3}}
$$

where $\boldsymbol{y}=\left(y_{1}, y_{2}, y_{3}\right), y_{l} \in\{0, \ldots, n\}$ for $l=1,2,3, \sum_{l=1}^{3} y_{l}=n$, and

$$
\begin{aligned}
& \theta_{1}(\phi)=\phi \sum_{i=1}^{m} p_{i}^{3}+(1-\phi) \sum_{i=1}^{m} p_{i}^{2} ; \\
& \theta_{2}(\phi)=3 \phi \sum_{i=1}^{m} \sum_{j=1}^{m} p_{i}^{2} p_{j}+(1-\phi) \sum_{i=1}^{m} \sum_{j=1}^{m} p_{i} p_{j}, \quad \text { for } \quad i \neq j ; \\
& \theta_{3}(\phi)=\phi \sum_{i=1}^{m} \sum_{j=1}^{m} \sum_{k=1}^{m} p_{i} p_{j} p_{k}, \quad \text { for } \quad i \neq j \neq k
\end{aligned}
$$

Notice that $\theta_{l}(\phi)$ can be interpreted as the probability of being $l, l=1,2,3$, the number of fragmented peaks to be observed in the microsatellite locus of interest. It can also be observed that $\sum_{l=1}^{3} \theta_{l}(\phi)=\phi\left(\sum_{i=1}^{m} p_{i}\right)^{3}+(1-\phi)\left(\sum_{i=1}^{m} p_{i}\right)^{2}=1$.

\subsection{Reference analysis}

The use of non-informative priors can be useful to study rare aneuploidies, for instance, in which little initial information about $\phi$ is available.

There are several methods to obtain reference or non-informative priors. Such methods depend on the concept of information that is under consideration. The simplest way to obtain a non-informative opinion about the parameter is to declare a uniform distribution as the measure of the initial uncertainty. However, uniform distribution can be an improper prior, if the range of the parameter is unbounded, and it is not invariant to a one-to-one transformation (see Migon and Gamerman (1999), (Bernardo and Smith (1994) and many others).

A class of non-informative priors which are invariant under transformations is introduced by Jeffreys (1961). In spite of solving the invariance problem, Jeffreys's prior - which, under some regularity conditions, is the reference prior introduced by Bernardo (1979) - can also be improper. Jeffreys's prior depends exclusively on Fisher's information. Consequently, it put more mass for values of $\phi$ for which data bring more information. The idea behind Jeffreys's prior comes from the desire of making inference based on a minimum of subjective previous information. It should be used in situations where there is little or no initial information or, if it does exist, we do not wish to use it, thus yielding a neutral analysis.

In this section we obtain Jeffreys's prior for $\phi$ and prove that it is a proper distribution. Notice that for the problem considered here Jeffreys's prior is easily obtained.

From (1) we obtain that Fisher's information is given by:

$$
I(\phi)=n\left(\frac{a^{2}}{\theta_{1}(\phi)}+\frac{b^{2}}{\theta_{2}(\phi)}+\frac{c^{2}}{\theta_{3}(\phi)}\right),
$$


in $\quad$ which $\quad a=\sum_{i=1}^{m} p_{i}^{3}-\sum_{i=1}^{m} p_{i}^{2}, \quad b=\sum_{i=1}^{m} \sum_{j=1}^{m} p_{i}^{2} p_{j}-\sum_{i=1}^{m} \sum_{j=1}^{m} p_{i} p_{j}, \quad$ for $\quad i \neq j, \quad$ and $\quad c=$ $\sum_{i=1}^{m} \sum_{j=1}^{m} \sum_{k=1}^{m} p_{i} p_{j} p_{k}$, for $i \neq j \neq k$. Consequently, Jeffreys's prior for $\phi$ is:

$$
\pi(\phi) \propto\left(\frac{a^{2}}{\theta_{1}(\phi)}+\frac{b^{2}}{\theta_{2}(\phi)}+\frac{c}{\phi}\right)^{1 / 2} .
$$

Proposition 1 Let $p_{i} \in[0,1]$, for all $i=1 \ldots, m$, and assume that $p_{i} \neq 0$ for any $i$. Then, Jeffreys's prior given in (2) is a proper distribution.

Proof Notice that

$$
\begin{aligned}
& \lim _{\phi \rightarrow 0} \frac{a^{2}}{a \phi+\sum_{i=1}^{m} p_{i}^{2}}=\frac{a^{2}}{\sum_{i=1}^{m} p_{i}^{2}} \\
& \lim _{\phi \rightarrow 0} \frac{b^{2}}{b \phi+\sum_{i=1}^{m} \sum_{j=1}^{m} p_{i} p_{j}}=\frac{b^{2}}{\sum_{i=1}^{m} \sum_{j=1}^{m} p_{i} p_{j}} \\
& \lim _{\phi \rightarrow 0} \frac{c}{\phi}=\infty .
\end{aligned}
$$

Consequently, there is $k \in(0,1)$ such that $a^{2}\left[a \phi+\sum_{i=1}^{m} p_{i}^{2}\right]^{-1}<c \phi^{-1}$ and $b^{2}\left[b \phi+\sum_{i=1}^{m} \sum_{j=1}^{m} p_{i} p_{j}\right]^{-1}$ $<c \phi^{-1}$ for all $\phi \in[0, k]$. Thus, it follows that:

$$
\int_{0}^{k} \pi(\phi) \mathrm{d} \phi<\int_{0}^{k}\left(\frac{3 c}{\phi}\right)^{1 / 2} \mathrm{~d} \phi=2(3 c k)^{1 / 2} .
$$

It is also noticeable that, for all $\phi \in(k, 1], \pi(\phi)<(3 M)^{1 / 2}$, in which $M=\max \left\{a^{2}\left[a \phi+\sum_{i=1}^{m} p_{i}^{2}\right]^{-1}\right.$, $\left.b^{2}\left[b \phi+\sum_{i=1}^{m} \sum_{j=1}^{m} p_{i} p_{j}\right]^{-1}, c \phi^{-1}\right\}+1$. Concluding the proof.

From (1) and (2) it follows that the posterior distribution of $\phi$ is given by:

$$
\pi(\phi \mid \boldsymbol{Y})=\frac{\frac{n !}{y_{1} ! y_{2} ! y_{3} !}\left[\theta_{1}(\phi)\right]^{y_{1}}\left[\theta_{2}(\phi)\right]^{y_{2}}\left[\theta_{3}(\phi)\right]^{y_{3}}\left(\frac{a^{2}}{\theta_{1}(\phi)}+\frac{b^{2}}{\theta_{2}(\phi)}+\frac{c}{\phi}\right)^{1 / 2}}{\int_{0}^{1} \frac{n !}{y_{1} ! y_{2} ! y_{3} !}\left[\theta_{1}(\phi)\right]^{y_{1}}\left[\theta_{2}(\phi)\right]^{y_{2}}\left[\theta_{3}(\phi)\right]^{y_{3}}\left(\frac{a^{2}}{\theta_{1}(\phi)}+\frac{b^{2}}{\theta_{2}(\phi)}+\frac{c}{\phi}\right)^{1 / 2} \mathrm{~d} \phi} .
$$

As a consequence of Proposition 1, the posterior distribution of $\phi$ given in (3) is proper. It can also be noticed that $\pi(\phi \mid \boldsymbol{Y})$ can not be obtained analytically.

\subsection{Bayesian procedures for hypothesis test for $\phi$}

Suppose that we are interested in a precise null hypothesis for $\phi$, that is, consider the following test:

$$
H_{0}: \phi \in \Phi_{0} \quad \text { versus } H_{1}: \phi \notin \Phi_{0},
$$

in which $\Phi_{0}=\left\{\phi_{0}\right\} \subset \Phi, \phi_{0} \in[0,1]$ is a known value and $\Phi$ is the parametric space of $\phi$.

Typically, from Bayesian point-of-view, we elicit prior probabilities $P\left(H_{i}\right)$ for the hypotheses $H_{i}$, $i=0,1$, and accept $H_{0}$ whenever its posterior probability, computed through Bayes's theorem, is larger than the posterior probability of $H_{1}$. Moreover, assuming that $P\left(H_{0}\right)=P\left(H_{1}\right)$, there is evidence in favour of $H_{0}$ if the Bayes factor (BF) is larger than one. For a detailed explanation of BF, see Bernardo and Smith (1994), Migon and Gamerman (1999) and many others.

For the problem under consideration, assuming that the prior distribution for $\phi$ is $\pi(\phi)$, the Bayes factor for the hypothesis in (4) is given by:

$$
\operatorname{BF}\left(H_{0}, H_{1}\right)=\frac{\left[\theta_{1}\left(\phi_{0}\right)\right]^{y_{1}}\left[\theta_{2}\left(\phi_{0}\right)\right]^{y_{2}}\left[\theta_{3}\left(\phi_{0}\right)\right]^{y_{3}} \int_{0}^{1} \pi(\phi) \mathrm{d} \phi}{\int_{0}^{1}\left[\theta_{1}(\phi)\right]^{y_{1}}\left[\theta_{2}(\phi)\right]^{y_{2}}\left[\theta_{3}(\phi)\right]^{y_{3}} \pi(\phi) \mathrm{d} \phi} .
$$


It is well known that, for testing precise hypothesis, BF can lead to Lindley's paradox (Tsao, 2006). FBST - briefly described in next section - was introduced in literature in order to avoid such a problem.

\subsubsection{Full Bayesian significance test (FBST) for $\phi$}

FBST (Pereira and Stern 1999, 2001) does not introduce prior probabilities for the hypotheses $H_{i}$ and makes the test for precise hypotheses simple. To perform FBST, the only necessary information is the posterior distribution for $\phi$. In this case, $H_{0}$ is accepted if $\Phi_{0}$ is in a high posterior probability region of $\Phi$. FBST in its invariant formulation due to Madruag et al. (2003) is defined as follows.

Let $\pi(\phi \mid \mathbf{y})$ be the posterior density of $\phi$. Denote by $r(\phi)$ a reference density on $\Phi$. Consider the following highest relative surprise set (HRSS):

$$
T(\boldsymbol{y})=\left\{\phi \in \Phi: \frac{\pi(\phi \mid \mathbf{y})}{r(\phi)} \geq \sup _{\Phi_{0}}\left\{\frac{\pi(\phi \mid \mathbf{y})}{r(\phi)}\right\}\right\} .
$$

The evidence in favour of the null hypothesis is given by $\operatorname{EV}\left(H_{0}, y\right)=1-\operatorname{Pr}(\phi \in T(\boldsymbol{y}) \mid \boldsymbol{y})$. The null hypothesis $H_{0}$ is accepted whenever $\operatorname{EV}\left(H_{0}, \boldsymbol{y}\right)$ is large. Madruga et al. (2003) prove that the evidence in favour of $H_{0}$ is invariant with respect to proper reparametrizations. A discussion about the "Bayesianity" of FBST can be found in Madruga et al. (2001).

For the fraction of non-disjunction in meiosis I, we will only consider the precise hypothesis in (4). We assume that the reference density is the uniform distribution. Consequenly, the HRSS $T(\boldsymbol{y})$ reduces to $\left\{\phi \in \Phi: \pi(\phi \mid \boldsymbol{y})>\pi\left(\phi_{0} \mid \boldsymbol{y}\right)\right\}$.

\subsection{Computational procedures}

Since the posterior distribution can not be obtained analytically, we consider a Newton-Cotes type method, named Simpson rule (Migon and Gamerman, 1999), to approximate the integrals in Eq. (3) and (5).

For computing the evidence of the null hypothesis, we divide the interval $(0,1)$ into a large number $k$ of subintervals, that is, we consider $\left(0, \frac{1}{k}\right],\left(\frac{1}{k}, \frac{2}{k}\right], \ldots,\left(\frac{k-1}{k}, 1\right)$ and evaluate the function

$$
\hat{\boldsymbol{\pi}}(\phi \mid \boldsymbol{y})=\frac{\left[\theta_{1}(\phi)\right]^{y_{1}}\left[\theta_{2}(\phi)\right]^{y_{2}}\left[\theta_{3}(\phi)\right]^{y_{3}}\left(\frac{a^{2}}{\theta_{1}(\phi)}+\frac{b^{2}}{\theta_{2}(\phi)}+\frac{c}{\phi}\right)^{1 / 2}}{\hat{\boldsymbol{I}}_{p}}
$$

at each endpoint of such intervals, in which $\hat{I}_{p}$ denotes the value provided by Simpson rule to the integral in (3).

The evidence of the null hypothesis can be obtained by noticing that $\pi\left(\phi_{0} \mid \boldsymbol{y}\right)$ can be estimated by (6) and, consequently, the evidence of $H_{0}$ is approximated by:

$$
\mathrm{EV}\left(H_{0}, \boldsymbol{y}\right)=1-\frac{\sum_{i=1}^{k-1} 1\left\{\hat{\boldsymbol{\pi}}\left(\frac{i}{k} \mid \boldsymbol{y}\right) \geq \hat{\boldsymbol{\pi}}\left(\phi_{0} \mid \boldsymbol{y}\right)\right\}}{k},
$$

in which $1\{A\}$ denotes the indicator function of $A$. Posterior means and modes and BF are computed in the regular way.

If we assume the uniform distribution to describe the prior uncertainty about $\phi$, we can obtain the posterior information for $\phi$ similarly.

\section{Simulation Studies: Test and Estimates of $\phi$}

In this section, we perform Monte Carlo studies in order to compare Bayes estimates of $\phi$ and the two Bayesian procedures for testing hypotheses about $\phi$ described in Section 2. Following Loschi and Franco (2006) we assume a microsatellite which presents six alleles with frequencies $0.12,0.45,0.09$, 
$0.31,0.01$ and 0.02 . This choice is motivated by the real application presented in Section 4 . We consider two non-informative prior specifications to describe the uncertainty about $\phi$ : Jeffreys's and uniform priors. For the problem considered here, Jeffreys's prior is more informative and tends to put most of its mass in small $\phi$ values.

Following Loschi and Franco (2006), for the SIR procedure we generate an initial sample of $\phi$ of size $T=100000$ and consider a posterior sample of size $S=5000$. We assume two sample sizes: $n=10$ and 100. Samples of size $n=10$ are included in the study to evaluate the efficiency of the estimators and test procedures in rare trissomies. Severous values of $\phi$ are considered.

\subsection{A comparison of Bayes estimates}

Tables 1 and 2 present some descriptive statistics for the posterior estimates of $\phi$ assuming sampling of sizes $n=10$ and $n=100$, respectively. We consider 500 replications from the multinomial distribution given in (1) with parameters $\phi=0.01,0.10,0.5,0.90$ and 0.99 .

From Table 1, in average, we notice that, for $n=10$, mode-Uniform always provides better estimates than mode-Jeffreys and that mean-Jeffreys is better than mean-Uniform for values of $\phi$ smaller than 0.50. In general, the posterior modes are closer to the real $\phi$ than the posterior means, except for $\phi=0.50$ and if the uniform prior distribution is assumed. If the uniform distribution is assumed, it is also noticeable that the posterior means and modes are higher than we obtain for Jeffreys's prior. For $\phi=0.01$ and $\phi=0.10$ we notice that, in general, the posterior means tend to overestimate the parameter and that the posterior modes tend to underestimate it. In these cases, it is noticeable that, at least, $75 \%$ of mode-Jeffreys and mode-Uniform are 0.0001 while, at least $75 \%$ of mean-Jeffreys (mean-Uniform) are higher than $0.1217(0.2303)$. For such values of $\phi$ we observe that the distributions of the estimates present many atypical observations (box-plots not shown) which pull the means up. For

Table 1 Descriptive Statistics for the posterior estimates of $\phi, n=10$.

\begin{tabular}{lllllll}
\hline Real $\phi$ & Estimator & Mean & St. Dev. & Q1 & Median & Q3 \\
\hline 0.01 & mean-Jeffreys & 0.1496 & 0.0527 & 0.1217 & 0.1409 & 0.1661 \\
& mean-Uniform & 0.2654 & 0.0566 & 0.2303 & 0.2598 & 0.2961 \\
& mode-Jeffreys & 0.0061 & 0.0361 & 0.0001 & 0.0001 & 0.0001 \\
& mode-Uniform & 0.0101 & 0.0588 & 0.0001 & 0.0001 & 0.0001 \\
0.10 & mean-Jeffreys & 0.2114 & 0.1244 & 0.1217 & 0.1661 & 0.2438 \\
& mean-Uniform & 0.3155 & 0.1043 & 0.2303 & 0.2961 & 0.3882 \\
& mode-Jeffreys & 0.0625 & 0.1313 & 0.0001 & 0.0001 & 0.0001 \\
0.50 & mode-Uniform & 0.0942 & 0.1791 & 0.0001 & 0.0001 & 0.0001 \\
& mean-Jeffreys & 0.4531 & 0.1899 & 0.3446 & 0.4854 & 0.5899 \\
0.90 & mean-Uniform & 0.5130 & 0.1543 & 0.4065 & 0.5375 & 0.6217 \\
& mode-Jeffreys & 0.4066 & 0.3333 & 0.1611 & 0.3330 & 0.6270 \\
& mode-Uniform & 0.4737 & 0.3259 & 0.2769 & 0.4921 & 0.7518 \\
& mean-Jeffreys & 0.6239 & 0.1723 & 0.5434 & 0.6502 & 0.7549 \\
& mean-Uniform & 0.6561 & 0.1425 & 0.5788 & 0.6703 & 0.7644 \\
& mode-Jeffreys & 0.7239 & 0.3275 & 0.5031 & 0.8649 & 1.0000 \\
& mode-Uniform & 0.7603 & 0.2940 & 0.5694 & 0.8704 & 1.0000 \\
& mean-Jeffreys & 0.6542 & 0.1595 & 0.5899 & 0.6894 & 0.7841 \\
& mean-Uniform & 0.6816 & 0.1331 & 0.6217 & 0.7065 & 0.7916 \\
& mode-Jeffreys & 0.7765 & 0.3032 & 0.6247 & 0.8834 & 1.0000 \\
& mode-Uniform & 0.8084 & 0.2658 & 0.6816 & 0.8830 & 1.0000 \\
\hline
\end{tabular}


Table 2 Descriptive Statistics for the posterior estimates of $\phi, n=100$.

\begin{tabular}{lllllll}
\hline Real $\phi$ & Estimator & Mean & St. Dev. & Q1 & Median & Q3 \\
\hline 0.01 & mean-Jeffreys & 0.0263 & 0.0214 & 0.0136 & 0.0147 & 0.0469 \\
& mean-Uniform & 0.0457 & 0.0198 & 0.0334 & 0.0358 & 0.0616 \\
& mode-Jeffreys & 0.0067 & 0.0133 & 0.0001 & 0.0001 & 0.0161 \\
\multirow{3}{*}{0.10} & mode-Uniform & 0.0114 & 0.0202 & 0.0001 & 0.0001 & 0.0318 \\
& mean-Jeffreys & 0.1182 & 0.0598 & 0.0798 & 0.1140 & 0.1608 \\
& mean-Uniform & 0.1337 & 0.0585 & 0.0943 & 0.1282 & 0.1756 \\
& mode-Jeffreys & 0.0871 & 0.0599 & 0.0494 & 0.0841 & 0.1295 \\
0.50 & mode-Uniform & 0.1025 & 0.0605 & 0.0649 & 0.0993 & 0.1454 \\
& mean-Jeffreys & 0.5117 & 0.1119 & 0.4388 & 0.5120 & 0.5874 \\
& mean-Uniform & 0.5197 & 0.1096 & 0.4492 & 0.5202 & 0.5935 \\
0.90 & mode-Jeffreys & 0.4954 & 0.1182 & 0.4167 & 0.4976 & 0.5746 \\
& mode-Uniform & 0.5041 & 0.1158 & 0.4275 & 0.5052 & 0.5813 \\
& mean-Jeffreys & 0.8398 & 0.0728 & 0.8015 & 0.8510 & 0.8941 \\
& mean-Uniform & 0.8410 & 0.0714 & 0.8034 & 0.8518 & 0.8944 \\
0.99 & mode-Jeffreys & 0.8880 & 0.1043 & 0.8182 & 0.8992 & 1.0000 \\
& mode-Uniform & 0.8885 & 0.1025 & 0.8197 & 0.8988 & 0.9992 \\
& mean-Jeffreys & 0.8806 & 0.0562 & 0.8532 & 0.8948 & 0.9230 \\
& mean-Uniform & 0.8812 & 0.0553 & 0.8539 & 0.8949 & 0.9230 \\
& mode-Jeffreys & 0.9445 & 0.0790 & 0.8993 & 0.9934 & 1.0000 \\
& mode-Uniform & 0.9444 & 0.0781 & 0.8989 & 0.9902 & 1.0000 \\
\hline
\end{tabular}

$\phi=0.90$ and $\phi=0.99$ we notice that the posterior means and modes tend to underestimate the parameter. For $\phi=0.90$, for example, we observe that, at least, $50 \%$ of mean-Jeffreys (mode-Jeffreys) are smaller than $0.6502(0.8649)$ while for the mean-Uniform (mode-Uniform) the median is 0.6703 (0.8705).

We notice from Table 1 that, for $\phi=0.01$ and $\phi=0.10(\phi=0.90$ and $\phi=0.99)$, the distributions of the estimates present many atypical values (box-plots not shown) pulling the means up (down). For $\phi=0.50$ the posterior means are, in general, better. For example, in this case, we notice that $50 \%$ of mean-Jeffreys are in the interval $(0.34455 ; 0.58994)$ while for mode-Jeffreys we observe that $50 \%$ of the estimates are higher than 0.1611 and smaller than 0.627 .

As expected, the estimates are considerably improved if we consider large sample size (see Table 2, for $n=100$ ). Similar to what happened to $n=10$, in average, mode-Uniform tends to estimate the parameter better, except for $\phi=0.99$. The distribution of the posterior estimates are more concentrated around their means and their means and medians are close to the real value of $\phi$. For all values of $\phi$, mean-Jeffreys (mode-Jeffreys) and mean-Uniform (mode Uniform) have the same behavior, presenting similar medians, means and standard deviations.

\subsection{Comparing FBST and BF}

In this section we compare BF and FBST for testing precise hypothesis about $\phi$, that is, we consider $\Phi_{0}=\left\{\phi_{0}\right\}$.

Since we are performing a Monte Carlo study, to fairly compare the test procedures, we take into consideration the following aspects about hypotheses tests. Hypotheses tests are partitions from both the parametric space, which is defined by the problem, and the sample space, which is defined by the statistician. Consequently, the statistician should look for the best partition for the sample space. By 
best partition it is meant the partition which minimizes the expected error that can be produced by our decision. DeGroot (1989) suggests using a partition which minimizes linear combinations of type I $(\alpha)$ and type II $(\beta)$ errors. In order to eliminate further considerations, in this paper we look for partitions which minimize $\alpha+\beta$. The spaces associated to the statistics $\mathrm{BF}\left(H_{0}, H_{1}\right)$ and $\mathrm{EV}\left(H_{0}, \boldsymbol{y}\right)$ are partitioned as $[0, k) \cup(k, \infty)$ and $\left[0, k^{*}\right) \cup\left(k^{*}, 1\right]$, respectively. The null hypothesis is rejected if the statistic is less than the cut point - say, $k$ for the $\mathrm{BF}$ and $k^{*}$ for the evidence. The best procedure is the one for which $\alpha+\beta$ evaluated in the best partition is the minimum.

We consider 100 replications from the multinomial distribution given in (1) with parameters $\phi=\phi_{0}, 0.1,0.2, \ldots, 0.9$ and assume $\phi_{0}=0.01,0.10,0.25,0.50,0.75,0.90,0.99$. We also consider the cut points $k^{*}=0.1,0.2, \ldots, 0.9$ and $k=0.45,0.90, \ldots, 3.60,4.05$.

For each partition and both test procedures, we calculate $\alpha$ and $\beta$ as follows. The type I error $\alpha$ for the FBST is the proportion of samples for which we observe EV $\left(H_{0}, \boldsymbol{y}\right)<k^{*}$ whenever $\phi=\phi_{0}$. Similarly, we obtain $\alpha$ for the Bayes factor. The type II error can be obtained using some results from probability calculus. The type II error $\beta$ for the FBST is obtained, firstly, evaluating the proportion of samples satisfying $\mathrm{EV}\left(H_{0}, \boldsymbol{y}\right) \geq k^{*}$, for every $\phi \neq \phi_{0}$ and then taking the weighted average of these proportions using the prior density of $\phi$ to define the weights entering this averages. For the Bayes factor, assuming that $P\left(H_{0}\right)=p, p \in(0,1)$, the type II error is given by:

$$
\beta=\frac{1}{1-p}\left\{\int_{0}^{\phi_{0}} P\left(\mathrm{BF}\left(H_{0}, H_{1}\right) \geq k \mid \phi\right) \pi(\phi) \mathrm{d} \phi+\int_{\phi_{0}}^{1} P\left(\mathrm{BF}\left(H_{0}, H_{1}\right) \geq k \mid \phi\right) \pi(\phi) \mathrm{d} \phi\right\} .
$$

In the simulation study, we consider a discretization of the parametric space and also assume that the probability $1-p$ is uniformly distributed among all $\phi \neq \phi_{0}-$ as usually done in Bayes Factor approach for testing. Consequently, in order to obtain a convex linear combination of the probabilities $P\left(\mathrm{BF}\left(H_{0}, H_{1}\right) \geq k \mid \phi\right)$, we approximate $\beta$ by:

$$
\beta=\frac{1}{l} \sum_{\phi \neq \phi_{0}} P\left(\mathrm{BF}\left(H_{0}, H_{1}\right) \geq k \mid \phi\right)
$$

where $l$ is the number of points considered in the discretization of $(0,1)$ space.

Figures 2 and 3 show the average for the posterior evidences of $H_{0}$ and Bayes factors for uniform (solid line) and Jeffreys's (dashed line) priors for samples of size $n=10$ and $n=100$, respectively. The cut points for both test procedures are also plotted considering dotted lines for the uniform prior and dotdashed lines for Jeffreys's prior. The results are shown for $\phi_{0}=0.01,0.50$ and 0.99 only.

From Figure 2 we notice that, in average, FBST is less influenced by the prior specifications than Bayes factor, for both sample sizes. It is noticeable that the mean evidences for both prior specifications are close. As expected, for samples of size $n=100$ (see Figure 3), in general, the evidence in favour (whenever $\phi=\phi_{0}$ ) or against (whenever $\left.\phi \neq \phi_{0}\right) H_{0}$, giving by FBST as well as BF, tends to be stronger than it was observed for $n=10$.

Particularly, for samples of size $n=10$, in average, we notice that FBST and BF lead to the same decision for $\phi_{0}=0.99$ and both prior specifications rejecting the null hypothesis for $\phi$ equal to 0.1 , $0.2,0.3$ and 0.4. For $\phi_{0}=0.01$, we observe that FBST and BF lead to different decisions for some values of $\phi$ and also that the same test procedure can yield different result if different priors are assumed. Notice, for instance, that if we consider the uniform distribution, FBST leads to the rejection of $H_{0}$ for $\phi=0.5, \ldots, 0.9$ while BF leads to the rejection of $H_{0}$ for $\phi=0.7,0.8,0.9$. In this case, we can also observe that, for Jeffreys's prior, FBST (BF) leads to the rejection of the null hypothesis if $\phi=0.7,0.8,0.9(\phi=0.6,0.7,0.8,0.9)$. Moreover, for $\phi_{0}=0.50$, we observe that $H_{0}$ is always accepted if we consider Jeffreys's prior and FBST or if we elicit the uniform prior and consider de BF to make decisions about $H_{0}$.

Comparing the two test procedures, from Figure 2 we notice that, in average, FBST (BF) is slightly better for $\phi=0.01,0.50$ and if the uniform (Jeffreys's) prior is assumed. In general, both test procedures tend to accept the null hypothesis for real values of $\phi$ close to $\phi_{0}$. 

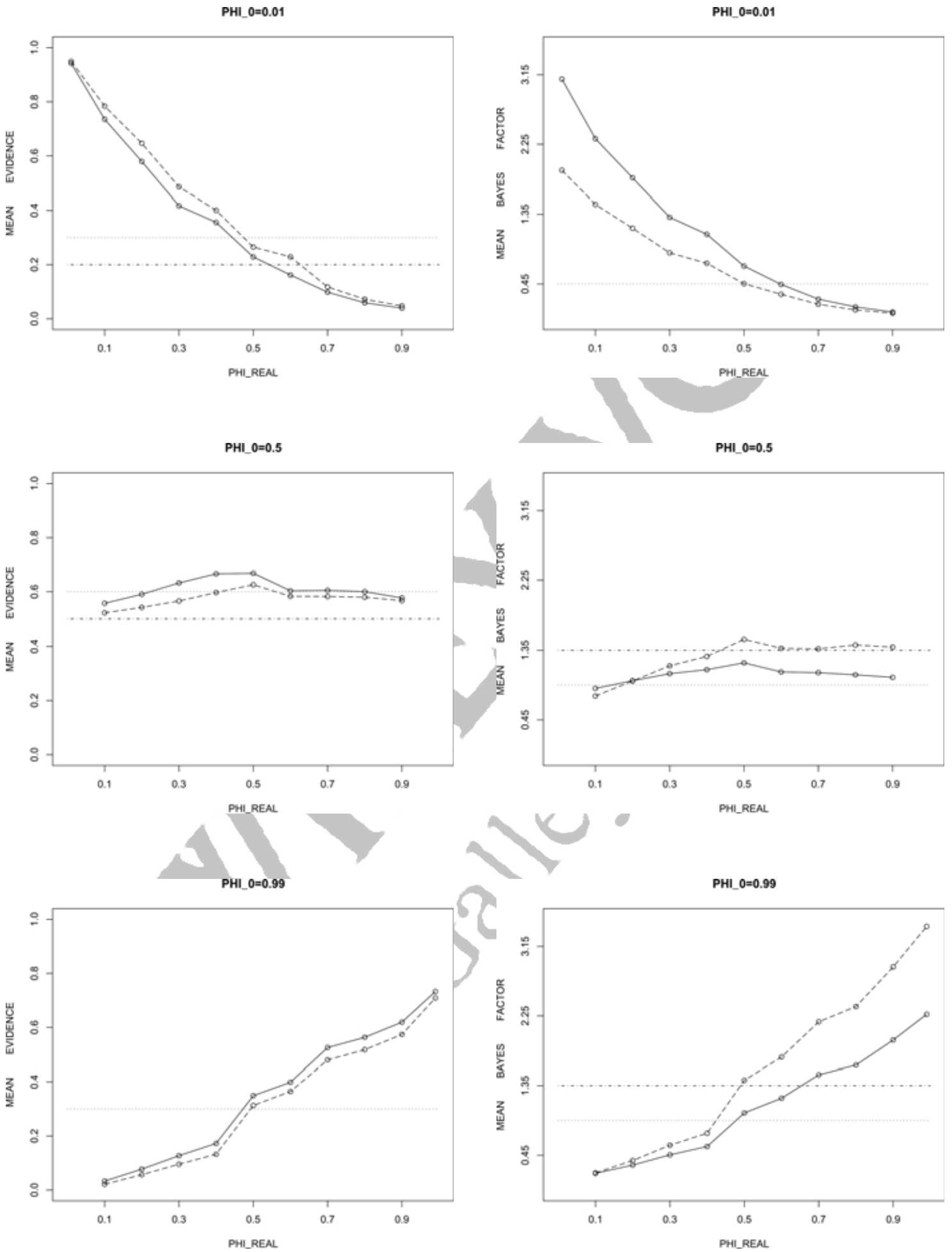

Figure 2 Mean evidence and Mean Bayes factor for uniform (solid line) and Jeffreys's (dashed line) priors, $n=10$, cut point-uniform (dotted line) and cut point-Jeffreys's (dotdashed line). 
1

2

3

4

5

6

7

8

9

10

11

12

13

14

15

16

17

18

19

20

21

22

23

24

25

26

27

28

29

30

31

32

33

34

35

36

37

38

39

40

41

42

43

44

45

46

47

48

49

50

51
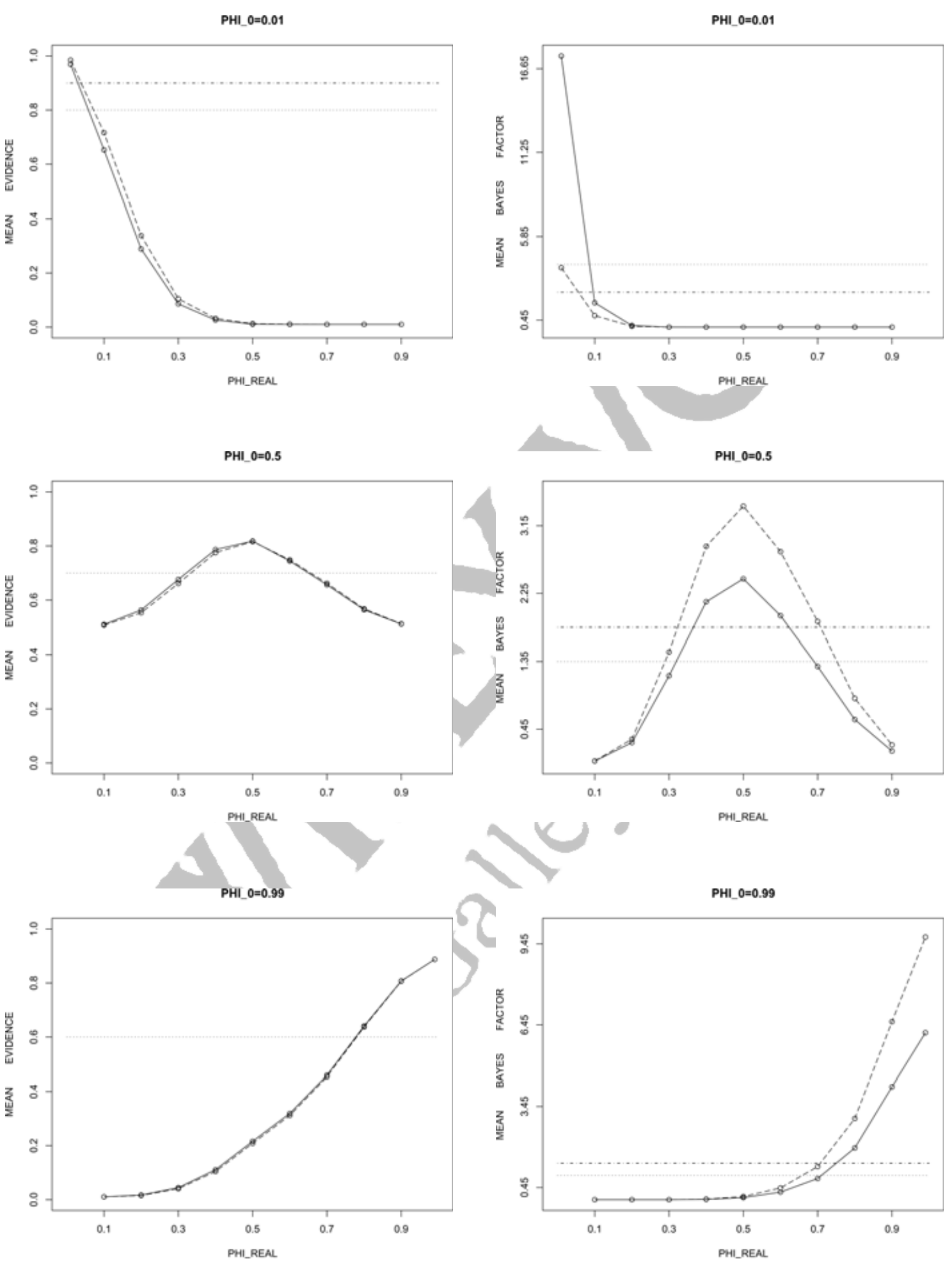

Figure 3 Mean evidence and Mean Bayes factor for uniform (solid line) and Jeffreys's (dashed line) priors, $n=100$, cut point-uniform (dotted line) and cut point-Jeffreys's (dotdashed line). 
Table 3 Best partition and errors for FBST and BF, $n=10$.

\begin{tabular}{|c|c|c|c|c|c|c|c|c|c|}
\hline \multirow[b]{2}{*}{$\phi_{0}$} & \multirow[b]{2}{*}{ Prior Distribution } & \multicolumn{4}{|c|}{ Evidence } & \multicolumn{4}{|c|}{ Bayes Factor } \\
\hline & & $\alpha$ & $\beta$ & $\alpha+\beta$ & $k^{*}$ & $\alpha$ & $\beta$ & $\alpha+\beta$ & $k$ \\
\hline \multirow[t]{2}{*}{0.01} & Uniform & 0.050 & 0.291 & 0.341 & 0.30 & 0.050 & 0.291 & 0.341 & 0.45 \\
\hline & Jeffreys & 0.030 & 0.358 & 0.388 & 0.20 & 0.050 & 0.291 & 0.341 & 0.45 \\
\hline \multirow[t]{2}{*}{0.10} & Uniform & 0.210 & 0.315 & 0.525 & 0.50 & 0.150 & 0.379 & 0.529 & 0.90 \\
\hline & Jeffreys & 0.090 & 0.305 & 0.395 & 0.60 & 0.150 & 0.379 & 0.529 & 0.90 \\
\hline \multirow[t]{2}{*}{0.25} & Uniform & 0.300 & 0.408 & 0.708 & 0.70 & 0.320 & 0.387 & 0.707 & 1.35 \\
\hline & Jeffreys & 0.200 & 0.483 & 0.683 & 0.70 & 0.190 & 0.622 & 0.812 & 0.90 \\
\hline \multirow[t]{2}{*}{0.50} & Uniform & 0.400 & 0.374 & 0.774 & 0.60 & 0.220 & 0.593 & 0.813 & 0.90 \\
\hline & Jeffreys & 0.360 & 0.378 & 0.738 & 0.50 & 0.210 & 0.558 & 0.768 & 1.35 \\
\hline \multirow[t]{2}{*}{0.75} & Uniform & 0.280 & 0.411 & 0.691 & 0.60 & 0.360 & 0.337 & 0.697 & 1.35 \\
\hline & Jeffreys & 0.340 & 0.298 & 0.638 & 0.70 & 0.300 & 0.389 & 0.689 & 1.80 \\
\hline \multirow[t]{2}{*}{0.90} & Uniform & 0.180 & 0.319 & 0.499 & 0.50 & 0.120 & 0.426 & 0.546 & 0.90 \\
\hline & Jeffreys & 0.180 & 0.264 & 0.444 & 0.40 & 0.180 & 0.319 & 0.499 & 1.35 \\
\hline \multirow[t]{2}{*}{0.99} & Uniform & 0.150 & 0.358 & 0.508 & 0.30 & 0.150 & 0.358 & 0.508 & 0.90 \\
\hline & Jeffreys & 0.150 & 0.298 & 0.448 & 0.30 & 0.170 & 0.352 & 0.522 & 1.35 \\
\hline
\end{tabular}

Figure 3 shows the results for samples of size $n=100$. In average, FBST leads to the same conclusion for both prior specifications and for all values of $\phi_{0}$ considered in the analysis. Although the mean BF is quite different for Jeffreys's and uniform priors - what is not observed by the FBST the results obtained using such priors are basically the same being different only for $\phi_{0}=0.50$. In this case, we notice that BF leads to the rejection of $H_{0}$ if the uniform prior is assumed, for $\phi=0.7$ only. That is, FBST and BF lead to similar conclusions about $H_{0}$ for $\phi_{0}=0.01$ and 0.99 as well as for $\phi_{0}=0.50$ whenever the uniform prior is assumed as prior specification.

Tables 3 and 4 provide the best partition and type I and type II errors associated to BF and FBST for samples of size $n=10$ and 100 , respectively. Type I and type II errors are obtained through simulation.

For samples of size $n=10$, we notice from Table 3 that FBST, in general, has upper performance for both prior specifications (except for $\phi_{0}=0.01$ and considering Jeffreys's prior and for $\phi_{0}=0.25$ assuming the uniform prior). We notice that FBST and BF provide equal values for $\alpha+\beta$ if $\phi_{0}=0.01,0.99$ for the uniform prior. Table 4 shows that, for $n=100$, in general, FBST has upper performance for both prior specifications and for $\phi_{0}=0.50,0.75,0.90$ and 0.99 (except for $\phi_{0}=0.75$, if the uniform prior is under consideration). BF presents better results for small values of $\phi_{0}$ and for both priors (except for $\phi_{0}=0.10$ if we assume the Jeffreys's prior). In short, we can say that, for small sample sizes, FBST is, in general, better and for large sample sizes, FBST has upper performance for large values of $\phi_{0}$.

\section{Case Study: The Down Syndrome Data}

In order to illustrate the methodology presented in previous sections we analyze the data set reported in Franco et al. (2003) which consists of a random sample of blood from 34 Brazilian individuals with trisomy of chromosome 21. The trisomy of chromosome 21 produces the Down syndrome and, in humans, is the most common cause of mental retardation of genetic origin. For this data set, the observed numbers of patients with one, two and three peaks are 6,22 and 6, respectively. The hypoth- 
Table 4 Best partition and errors for FBST and BF, $n=100$.

\begin{tabular}{|c|c|c|c|c|c|c|c|c|c|}
\hline \multirow[b]{2}{*}{$\phi_{0}$} & \multirow[b]{2}{*}{ Prior Distribution } & \multicolumn{4}{|c|}{ Evidence } & \multicolumn{4}{|c|}{ Bayes Factor } \\
\hline & & $\alpha$ & $\beta$ & $\alpha+\beta$ & $k^{*}$ & $\alpha$ & $\beta$ & $\alpha+\beta$ & $k$ \\
\hline \multirow[t]{2}{*}{0.01} & Uniform & 0.010 & 0.033 & 0.043 & 0.80 & 0.020 & 0.021 & 0.041 & 4.05 \\
\hline & Jeffreys & 0.020 & 0.036 & 0.056 & 0.90 & 0.020 & 0.021 & 0.041 & 2.25 \\
\hline \multirow[t]{2}{*}{0.10} & Uniform & 0.020 & 0.114 & 0.134 & 0.70 & 0.010 & 0.116 & 0.126 & 0.90 \\
\hline & Jeffreys & 0.010 & 0.149 & 0.159 & 0.70 & 0.090 & 0.121 & 0.211 & 0.90 \\
\hline \multirow[t]{2}{*}{0.25} & Uniform & 0.080 & 0.372 & 0.452 & 0.70 & 0.070 & 0.347 & 0.417 & 0.90 \\
\hline & Jeffreys & 0.250 & 0.289 & 0.539 & 0.80 & 0.180 & 0.409 & 0.439 & 0.45 \\
\hline \multirow[t]{2}{*}{0.50} & Uniform & 0.200 & 0.286 & 0.486 & 0.70 & 0.200 & 0.290 & 0.490 & 1.35 \\
\hline & Jeffreys & 0.220 & 0.252 & 0.472 & 0.70 & 0.190 & 0.299 & 0.489 & 1.80 \\
\hline \multirow[t]{2}{*}{0.75} & Uniform & 0.100 & 0.387 & 0.487 & 0.70 & 0.130 & 0.341 & 0.471 & 1.35 \\
\hline & Jeffreys & 0.100 & 0.308 & 0.408 & 0.70 & 0.140 & 0.319 & 0.459 & 2.25 \\
\hline \multirow[t]{2}{*}{0.90} & Uniform & 0.030 & 0.213 & 0.243 & 0.60 & 0.080 & 0.165 & 0.328 & 1.35 \\
\hline & Jeffreys & 0.030 & 0.165 & 0.195 & 0.60 & 0.030 & 0.159 & 0.239 & 2.25 \\
\hline \multirow[t]{2}{*}{0.99} & Uniform & 0.040 & 0.203 & 0.243 & 0.60 & 0.040 & 0.204 & 0.244 & 1.35 \\
\hline & Jeffreys & 0.040 & & 0.199 & 0.60 & 0.040 & 0.207 & 0.247 & 1.35 \\
\hline
\end{tabular}

esis of Hardy-Weinberg equilibrium is verified for the Brazilian population and six alleles are found with frequencies $0.12,0.45,0.09,0.31,0.01$ and 0.02 .

The maximum likelihood estimates for $\phi$ and its asymptotic variance obtained by Franco et al. (2003) are 0.6552 and 0.0481 , respectively.

Table 5 shows the results obtained by considering Jeffreys's and uniform prior distributions and the procedures presented in previous sections.

It is noticeable from Table 5 that the posterior estimates using Jeffreys's and uniform priors are similar and that, for each prior distribution, mode and mean provide very close estimates. We also perceived that, if the uniform distribution is assumed to describe the initial uncertainty on $\phi$, the posterior mean and mode are close to the maximum likelihood estimate obtained by Franco et al. (2003). It is noticeable from Figure 4 that both posterior distributions put substantial mass around 0.65, they are asymmetric distributions and have unique modes. (See Loschi and Franco (2006) for the analysis using some informative prior distributions).

Bootstrap confidence interval was considered by Barros and Franco (2002) for testing if the fraction $\phi$ of non-disjunction in Meiosis I for patients with Down syndrome in the Brazilian population is 0.68 . Such procedure leads to accept the null hypothesis. We test the same hypotheses (say, $H_{0}: \phi=0.68$ versus $\left.H_{a}: \phi \neq 0.68\right)$ using the Bayesian procedures for testing hypotheses introduced

Table 5 Posterior estimates for $\phi$, Brazilian Down syndrome patients.

\begin{tabular}{llll}
\hline Prior Distributions & \multicolumn{3}{c}{ Posterior Summaries } \\
\cline { 2 - 4 } & Mean & Variance & Mode \\
\hline Jeffreys's & 0.6417 & 0.0322 & 0.6374 \\
Uniform & 0.6549 & 0.0305 & 0.6552 \\
\hline
\end{tabular}




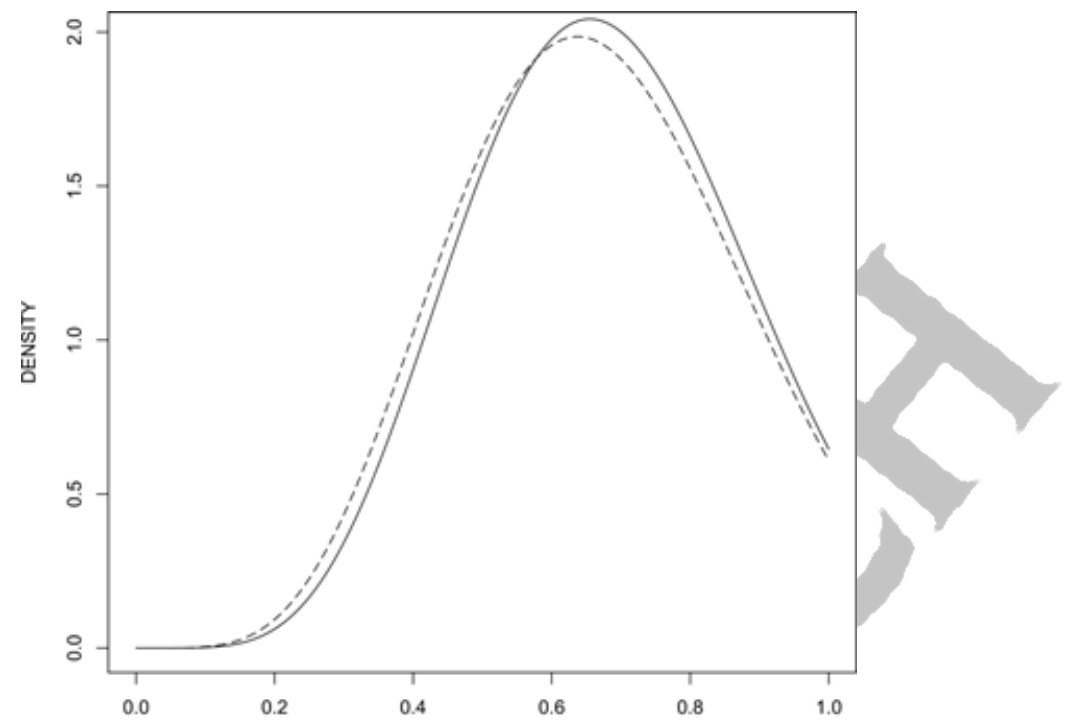

Figure 4 Posterior Distributions of $\phi$ for uniform (solid line) and Jeffreys's (dashed line) priors, Brazilian Down syndrome patients.

Table 6 Bayes factor and evidence of null $\mathrm{Hy}$ pothesis, Brazilian Down syndrome patients.

\begin{tabular}{lll}
\hline Prior specifications & Evidence & Bayes factor \\
\hline Jeffreys's & 0.9158 & 2.997 \\
Uniform & 0.9506 & 2.0290 \\
\hline & &
\end{tabular}

in previous sections. We notice from Table 6 that the evidence of the null hypothesis as well as Bayes factor are quite similar for both prior specifications. They assume very high values which means that both test procedures lead to accept that $\phi$ is 0.68 for the Brazilian population. Notice that these results are in agreement with Barros and Franco (2002) conclusions.

\section{Conclusions}

In this paper we considered Jeffreys's approach to obtain a non-informative prior (Jeffreys's prior) for the fraction $\phi$ of non disjunction in meiosis I in the absence of parental information. We proved that such distribution is proper. Posterior estimates of $\phi$ obtained using Jeffreys's and uniform priors were compared through a Monte Carlo study. Two Bayesian procedures for testing hypotheses about $\phi$ were introduced: full Bayesian significance test (FBST) and Bayes factor (BF). From the Bayesian point of view, BF is by far the most used procedure for testing hypothesis. However, it can lead to Lindley's paradox. FBST was introduced in literature as an alternative procedure for testing hypotheses and aims at avoiding such a problem. For this reason, a Monte Carlo study was performed in order to compare the efficiency of BF and FBST for testing precise hypothesis about $\phi$. We also analyzed 34 Brazilian patients with trisomy in the chromosome 21 using uniform and Jeffreys's priors. 
We noticed that the posterior estimates are similar if we consider as prior specifications both, uniform and Jeffreys's priors. Posterior modes, in general, provide better estimates for $\phi$ and these estimates are even better if the uniform distribution is assumed to describe the uncertainty about $\phi$. As expected, the posterior results are improved if we consider large sample size. Comparing FBST and $\mathrm{BF}$ for testing precise hypothesis about $\phi$ we notice that FBST is, in general, better for both prior specifications and both sample sizes. BF shows to be slightly better only for large sample sizes and small values of $\phi_{0}$.

For the Brazilian patients with Down syndrome, we noticed that the estimates for $\phi$, the evidence of the null hypotesis and Bayes factor are quite similar for both prior specifications. It was observed that the posterior mean and mode are close to the maximum likelihood estimate obtained by Franco et al. (2003), if the uniform distribution is assumed. We also observed that FBST and BF lead to accept that fraction of non-disjunction in meiosis $\mathrm{I}$ is $\phi=0.68$ for the Brazilian population which is in agreement with Barros and Franco (2002) statements.

Acknowledgements The authors would like to thank Professor Sergio D. J. Pena and Flavia C. Parra (UFMG) for providing the data set, Glaura C. Franco for comments and Alisson Duarte for the art. We also must express our gratitude to the Associate Editor and two referees whose constructive criticisms, maily in the presentation of the problem and in how to fairly compare the tests, led us to improve the paper substantially. R. H. Loschi acknowledges CNPq (Conselho Nacional de Desenvolvimento Científico e Tecnológico) of the Ministry for Science and Technology of Brazil (grants 304505/2006-4, 472877/2006-2, 472066/2004-8) for a partial allowance to her research. J. V. D. Monteiro, G. H. M. A. Rocha and V. D. Mayrink received financial support from CNPq via PIBIC program.

\section{References}

Barros, P. A. and Franco, G. C. (2002). Testes bootstrap para a fração de não disjunção meiótica em pacientes com síndrome de Down (in portuguese). Technical Report RTP-01/2002. Departament of Statistics. Universidade Federal de Minas Gerais. (URL: http://www.est.ufmg.br/rts/\#pes02)

Bernardo, J. M. (1979). Reference posterior distributions for Bayesian inference. Journal of the Royal Statistical Society B 41, 113-147.

Bernardo, J. M. and Smith, A. F. M. (1994). Bayesian Theory (1st edn). Wiley, Chichester.

DeGroot, M. H. (1989). Probability and Statistics (2nd edn). Addison Wesley, USA.

Franco, G. C., Lucio, P. S., Parra, F. C., and Pena, S. D. J. (2003). A probability model for the meiosis I non-disjunction fraction in numerical chromosomal anomalies. Statistics in Medicine 22(12), 2015-2024.

Griffin, D. K. (1996). The incidence, origin, and etiology of aneuploidy. International Review of Cytology 167, 64-70.

Hartl, D. L. and Clark, A. G. (1997). Principles of population in genetics (3rd. edition). Sunderland: Sinauer Associates.

Hassold, T. J. and Hunt, P. (2001). To er (meiotically) is human: the genesis of human aneuploidy. Nature Reviews in Genetics 2, 280-291.

Hassold, T. J. and Jacobs, P. A. (1984). Trisomy in man. Annual Reviews in Genetics 18, 69-97.

Jeffreys, H. (1961). Theory of Probability. Oxford, Claredon Press.

Koehler, K. E., Hawley, R. S., Sherman, S., and Hassold, T. (1996). Recombination and nondisjunction in human and flies. Human Molecular Genetics 5, 1495-1505.

Loschi, R. H. and Franco, G. C. (2006). Bayesian analysis for the meiosis i non-disjunction fraction in numerical chromosomal anomalies. Biometrical Journal 48(2), 220-232.

Madruga, M. R., Esteves, L. G., and Wechsler, S. (2001). On the Bayesianity of Pereira-Stern tests. Test 10(1), 291-299.

Madruga, M. R., Pereira, C. A. B., and Stern, J. M. (2003). Bayesian evidency test for precise hypotheses. Journal of Statistical Planning and Inference 117, 185-198.

Migon, H. S. and Gamerman, D. (1999). Statistical Inference: An integrated approach. New York: Arnold.

Nicolaidis, P. and Petersen, M. B. (1998). Origin and mechanisms of non-disjunction in human autosomal trisomies. Human Reproduction 13, 313-319. 
Pena, S. D. J. (1998). Molecular Cytogenetics I: PCR-based diagnosis of human trisomies using computer-assisted laser densitometry. Genetic Molecules Biology 3, 371-322.

Pereira, C. A. B. and Stern, J. M. (1999). Evidence and credibility: Full Bayesian significance test for precise hypotheses. Entropy 1, 69-80.

Pereira, C. A. B. and Stern, J. M. (2001). Model selection: Full Bayesian approach. Envirometrics 12, 559-568.

Petersen, M. B., Schinzel, A. A., Binkert, F., Tranebjaerg, L., Mikkelsen, M., Collins, F. A., Economou, C. P., and Antonarakis, S. E. (1992). Comparative study of micosatellite and cytogenetic markers for detecting the origin of the nondisjoined chromosome 21 in Down syndrome. American Journal of Human Genetic 167, 263-296.

Savage, A. R., Petersen, M. B., Pettay, B., Taft, L., Allran, K., Freeman, S. B., Karadina, G., Avramopoulos, D., Tofs, C., Mikkelsen, M., Hassold, T. J., and Sherman, S. L. (1998). Elucidating the mechanisms of parental non-disjunction of chromosome 21 in humans. Human Molecular Genetics 7, 1221-1227.

Tsao, C. A. (2006). A note on Lindley's paradox. Test 15(1), 125-139.

Yoon, P. W., Freeman, S. B., Sherman, S. L., Taft, L. F., Gu, Y., Pettay, D., Flanders, W. D., Khoury, M. J., and Hassold, T. J. (1996). Advanced maternal age and the risk of Down syndrome characterized by the meiotic stage of the chromosomal error: a population based study. American Journal of Human Genetics 58, 628633.

Zaragosa, M. V., Millie, E., Redline, R. W., and Hassold, T. J. (1994). Studies of non-disjunction in trisomies 2, 7, 15 and 22: does the parental origin of trisomy influence placental morphology? Journal of Medical Genetics 35, 924-931.

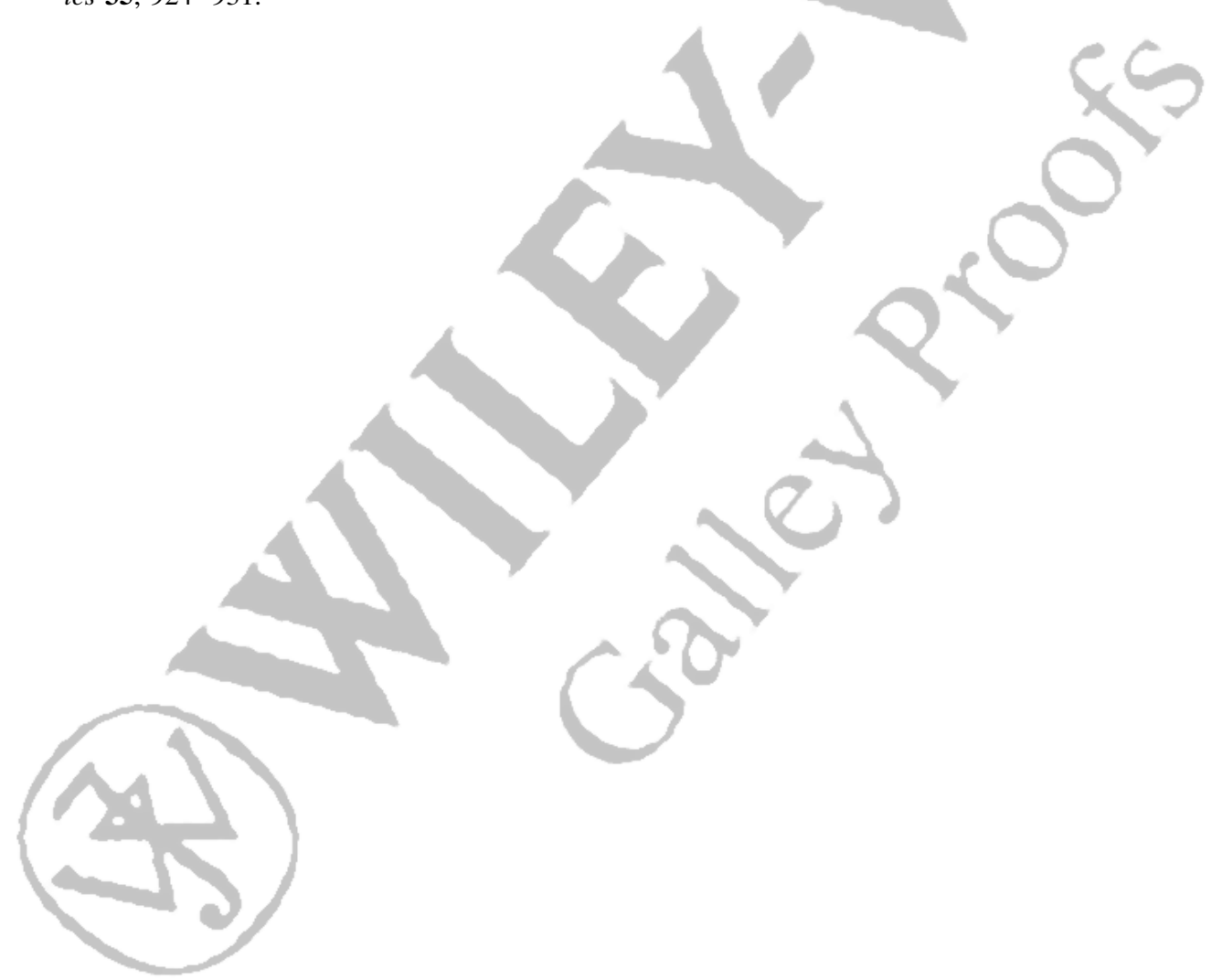

